\title{
PERSPECTIVA SUBJETIVA DE LA CALIDAD DE VIDA DEL ADULTO MAYOR, DIFERENCIAS LIGADAS AL GÉNERO Y A LA PRÁCTICA DE LA ACTIVIDAD FÍSICO RECREATIVA
}

\author{
Mónica Mora, Delia Villalobos, Gerardo Araya y Antonieta Ozols \\ Escuela Ciencias del Deporte \\ Facultad de Ciencias de la Salud \\ Universidad Nacional \\ mokimora@costarricense.cr
}

\section{RESUMEN}

\begin{abstract}
El propósito de este estudio fue conocer la relación entre las variables (soporte social, autonomía, salud mental, actividad físico recreativa) que integran la perspectiva subjetiva de la calidad de vida, ligadas al género en personas adultas mayores costarricenses. Además, conocer el principal motivo de estos sujetos para realizar la actividad física y el beneficio percibido por estos, de acuerdo al género. En este estudio participaron 152 sujetos (92 mujeres y 60 hombres) con edades entre los 60 a 75 años, de 21 centros diurnos, a los cuales se les aplicó dos instrumentos: un cuestionario de calidad de vida (INISA,1994) y una encuesta sobre actividad fisica recreativa. Los resultados mostraron relación significativa entre las variables autonomía y soporte social; autonomía y estado mental; autonomía y frecuencia de la actividad; autonomía y duración. Asimismo, en lo que respecta al género; la depresión geriátrica fue superior en las mujeres $(F=5.86)$. La salud fue el motivo principal para realizar la actividad física y los beneficios principales de ésta para ambos géneros fueron, el sentirse más reanimados, más alegres, con energía, y el alivio de dolores. Se concluye, que la calidad de vida (soporte social, autonomía, salud mental) percibida por el adulto mayor no difiere en lo que respecta al género e incluso en el motivo o beneficio para hacer actividad física, excepto en lo que respecta a la depresión, la cual se presenta con mayor frecuencia en las mujeres. Se comprueba que la actividad física recreativa le permite obtener al adulto mayor resultados positivos entre más cantidad de veces y de tiempo por semana le dedique.
\end{abstract}

PALABRAS CLAVES: calidad de vida, adulto mayor, perspectiva subjetiva, autonomía, salud mental, soporte social, actividad física.

\author{
SUBJECTIVE PERSPECTIVE OF THE QUALITY OF LIFE OF \\ SENIOR CITIZENS, DIFFERENCES RELATED TO GENDER AND \\ THE PRACTICE OF PHYSICAL RECREATIONAL ACTIVITIES
}

\begin{abstract}
The purpose of this study was to know the relationship between the variables (social support, autonomy, mental health, and physical recreational activity) included in the subjective perspective of the quality of life of Costa Rican senior citizens related to gender. Another objective was to know the main reason for the subjects to practice physical activity and the benefits perceived by them, according to their gender. A total of 152 subjects (92 women and 60 men) ranging between 60 and 75 years of age from 21 senior centers participated in this study. Two instruments were applied to the subjects: a questionnaire on quality of life (INISA, 1994) and a survey on physical recreational activity. Results showed a significant relationship between the variables autonomy and social support, autonomy and mental health, autonomy and frequency of activity, and autonomy and duration. Regarding gender, geriatric depression was more frequent in women $(F=5.86)$. Health was the main motivation to do physical activity and the major benefits for both genders were feeling more revitalized, happier, energized, and pain relief. In conclusion, quality of life (social support, autonomy, mental health) as perceived by senior citizens shows no differences as far as gender or reasons to exercise or benefits received, except for depression, which is more frequent in women. It is proven that the more times and the longer physical recreational activities are practiced by senior citizens per week the more positive results are received.
\end{abstract}

KEY WORDS: quality of life, senior citizens, subjective perspective, autonomy, mental health, social support, physical recreational activity. 


\section{INTRODUCCIÓN}

El aumento de la esperanza de vida en las sociedades avanzadas como consecuencia de la estabilidad y el bienestar alcanzado, ha ocasionado un cambio radical en las pirámides demográficas, produciéndose una transformación global (Ríos, Ríos y Padial, 2000).

Son muchas las personas en el mundo actual que dedican su esfuerzo y estudio a lo que podría denominarse como la nueva cultura de la longevidad. En realidad, es el intento de vivir más y en mejores condiciones físicas, sociales y mentales, producto de que el avance social está orientado hacia esa dirección, buscando así un modelo de envejecimiento competente en un sentido útil y productivo, capaz de fortalecer desde un punto de vista genérico de la salud su calidad de vida (Ríos y col, 2000). Se define la calidad de vida como un estado de bienestar; sin embargo esta noción tomará diferentes énfasis, según el contexto del cual parte su valoración (Faden y Germán, 1994).

Giusti (1991), define la calidad de vida como un estado de bienestar físico, social, emocional, espiritual, intelectual y ocupacional que le permite al individuo satisfacer apropiadamente sus necesidades individuales y colectivas. Para efectos de este estudio ese es el concepto operacional de calidad de vida. Además de estas consideraciones se toma como una variable importante el nivel de actividad física de los sujetos basándose en Abrante, Brito y García (1996); Santana (1991); García y col. (1990); entre otros. Como se puede observar, la calidad de vida es un complejo concepto cuya definición operacional y dimensional resulta francamente difícil. No obstante, hay acuerdo en cuanto a la necesidad de abordarla desde una dimensión subjetiva; es decir, lo que la persona valora de ella sin restringirla a la dimensión objetiva (opinión de otras personas o terceros) porque lo fundamental es la percepción propia (Rojas, 1999 y Rojas, 1997).

Autores como Díaz (1987), Faden y German, (1994), Hernández (2000) y Leturia (1998) visualizan la importancia de la valoración subjetiva en la calidad de vida. Sin embargo, usualmente las propuestas dirigidas a las personas mayores se orientan hacia una evaluación de los efectos que producen los medicamentos o la enfermedad. La perspectiva subjetiva de esta en el adulto mayor será el foco de atención de este estudio y para lo cuál se considerará la propuesta de Giusti (1991), INISA (1994) y Lawton (1991). De estos trabajos se considerarán cuatro variables (autonomía, soporte social, salud mental y actividad física recreativa) para delimitar operacionalmente la perspectiva subjetiva de la calidad de vida en este estudio.

Si se toma en cuenta las propuestas de los autores antes mencionados, las variables que involucran la perspectiva subjetiva de la calidad de vida se entenderán de la siguiente forma: autonomía estará definida como capacidad funcional, que se refiere a si puede o no realizar actividades básicas e instrumentales; el soporte social que se refiere a las estrategias (prestar ayuda, colaboración) que permiten relacionarse con los demás; salud mental el cual involucra el grado de armonía psicoemocional y la actividad física recreativa como la satisfacción y la disponibilidad de este para la realización de dichas actividades. Otros estudios recientemente han analizado los índices de calidad de vida, basados en una amplia gama de dimensiones y aspectos en los que se involucra la edad, el género y el nivel de actividad física entre las que se puede citar:

Fujisawa (1994), encontró diferencias en los índices de calidad de vida (apoyo social, autonomía, salud mental, relaciones familiares) con respecto al género. Roy y Fitz (1996), señalaron que con la edad avanzada los riesgos de tensión depositados en los adultos mayores por parte de la familia aumentan (su mortalidad) y recomiendan otros estudios que identifiquen predictores de mortalidad como el sedentarismo. 
Por su parte, Hernández (2000), señaló que se podría esperar diferencias en la calidad de vida de los adultos mayores ligadas a la edad. Sin embargo no se encontraron diferencias significativas en cuanto al género.

En el campo específico de Costa Rica algunas de las investigaciones realizadas en calidad de vida y adulto mayor es posible citar las desarrolladas por Céspedes y col. (1987), en la que se señalaron que existen carencias de tipo afectivo por falta de interacción social, contacto social y problemas de movilización bastante acentuados. Es decir, que los niveles de actividad física son importantes predictores de los índices de calidad de vida de este grupo etario.

Asimismo, Villalobos (1989) mostró que las personas mayores no cuentan con suficientes recursos económicos para satisfacer en forma adecuada sus necesidades básicas; en contraparte las que están institucionalizados, experimentan sentimientos de soledad por encontrarse separados de su núcleo familiar, mostrándose apáticos, poco participativos en las actividades programadas por los centros y tiene mayores factores de riesgo como sedentarismo y aislamiento. Además evidencian discapacidades más visibles y requieren mayor ayuda para realizar actividades de la vida diaria; en el caso de las personas mayores que vivían con su familia y eran atendidos en el centro diurno aportaron ayuda económica o de intervención en alguna tarea doméstica agrícola entre otras. Estos sujetos se mostraron más activos, participativos y se mantuvieron ocupados en diferentes tareas.

Agüero (1993), evidenció que el grupo sometido al programa de actividad física presentó una disminución de FC (frecuencia cardiaca) en reposo (3 lat/min) en comparación con el grupo control se mantuvo al mismo nivel; en cuanto a su PA (presión arterial) en reposo también se redujo hasta 9 $\mathrm{mm} / \mathrm{Hg}$ a diferencia del grupo control que fue únicamente de $2 \mathrm{~mm} / \mathrm{Hg}$ únicamente. Se desprende finalmente que la persona mayor que realiza actividad física, mejora su condición cardiovascular.

Aragón y Salas (1996), encontraron que los problemas físico-funcionales se relacionaron con la pérdida de fuerza muscular, flexibilidad, equilibrio, vista, memoria y audición, mientras que los problemas psicosociales respondieron a la pérdida de salud, tristeza, deterioro de las capacidades funcionales y discriminación familiar, entre otros.

Quirós (1996), en su estudio mostró que en las áreas urbanas los adultos mayores presentaron más autonomía, estimulación y proyectos de vida, por lo que se hace aún más necesario dicho modelo.

Por su parte Ureña y Delgado (1998), analizaron la relación entre ejercicio físico y la percepción subjetiva de la persona mayor en 90 adultos con edad promedio de 71.4 años; los resultados mostraron que el grupo de actividades acuáticas tenían una percepción más positiva de sí mismos, aunque difieren poco de los reportes del grupo de manualidades, caso contrario al presentado por el grupo control. El ejercicio físico implicado en las actividades acuáticas y la atmósfera que se genera en este contexto, visualizan positivamente esta actividad como opción importante para desarrollar una posición optimista en el adulto mayor.

Bolaños y Mora (1999), en su investigación sobre actividad física - recreativa y estado emocional en dos grupos gerontológicos del área central (San José - Heredia), encontraron que los sujetos participantes eran activos físicamente y su principal motivación es su estado de salud integral; asimismo se determinó que de acuerdo a la actividad física practicada, los estados emocionales resultan beneficiados y respecto al medio acuático se lograron determinar mejores sensaciones que el medio terrestre.

Rojas (1999), en su investigación sobre calidad de vida y autonomía en personas mayores, desde un enfoque cualitativo y en el cuál realizó un estudio de 8 casos de personas de 70 años (4 hombres y 4 
mujeres) y cuyo objetivo fue explorar la percepción de calidad de vida de estas, con base en su autonomía psicológica y conducta funcional. Los resultados mostraron que en general los adultos perciben un sentimiento de bienestar desde los diferentes ámbitos físico, social, espiritual, ocupacional, intelectual y psicoemocional; el cuál se fortalece por ser personas autónomas y funcionales.

El propósito fue conocer la relación entre las variables autonomía, soporte social, salud mental (depresión, estado mental) y actividad física (frecuencia semanal de actividad física recreativa, tiempo diario, disfrute de la actividad que realiza y la edad de los sujetos); considerando diferencias que se puedan presentar en esas relaciones por género. De esta manera, se analizará el efecto del género sobre las variables dependientes mencionadas. Además se pretende determinar cuál es el principal motivo para realizar la actividad física recreativa y cuáles son los beneficios que esta población presenta según el género.

\section{METODOLOGÍA}

Sujetos: en el estudio participaron de 92 mujeres y 60 hombres adultos mayores de 60 a 75 años de edad que asistían regularmente a 21 centros diurnos debidamente especificados como tal. La selección de los sujetos fue por el método aleatorio simple; utilizando una lista previa a la visita a cada centro. Con la tabla de números aleatorios se realizó la selección al azar de los sujetos del estudio en cuestión.

Instrumento: se tomó como base dos instrumentos; el primero es el cuestionario de calidad de vida elaborada en Costa Rica por el Instituto de Investigación en Salud (INISA), mediante el Programa de Investigación sobre el Envejecimiento en 1994 con una consistencia interna de 0.77, lo cual se considera apropiado estadísticamente (Thomas y Nelson, 1990). Este cuestionario se aplicó para medir las variables autonomía, soporte social y salud mental, parte del constructo calidad de vida y valoración subjetiva. Dicho instrumento constó de cinco partes dentro de las que se destacan: una primera de identificación, la cual consta de anotación de datos generales como nombre, lugar, el número de persona, fecha de la entrevista; se aplicó cada ítem igual que la versión final INISA (1994). Una segunda parte acerca de las características sociodemográficas en la que se indaga sobre su género, fecha de nacimiento, estado civil, grado de escolaridad alcanzado, tipo de pensión y seguro, la que no se tomó en cuenta debido a que no es un factor importante para efectos de este estudio, salvo el género y la fecha de nacimiento. Una tercera parte sobre soporte social en donde se pregunta acerca tiempo de radicar en la vivienda, número de personas que conviven en ésta, su parentesco, cuál es su desempeño dentro del seno familiar, participación en la comunidad y se tomó en cuenta solamente éstos dos últimos (ítems 14 y 15), los cuales permiten una mayor facilidad para cuantificar las respuestas de los sujetos. La cuarta parte de actividades del diario vivir en la que se le indaga al adulto mayor con preguntas cerradas sobre su alimentación, algunas actividades de higiene y cuido personal (y si requiere ayuda para ellas). Finalmente la quinta parte sobre salud mental donde se examina al adulto mayor en algunos rubros psicológicos básicos lo que permite descubrir su nivel mental y de depresión. Se aplicó completos todos los ítemes de estas dos últimas partes. En cuanto a la calificación, se le asignó un cero a la respuesta "NO" o negativa y un punto a la repuesta "SI" o afirmativa en cada uno de los ítems. Una excepción fue en la cuarta parte (salud mental) en donde cada ítem tenía un puntaje diferente, el cual osciló desde tres hasta seis puntos, dependiendo del número de preguntas por ítem. El segundo instrumento fue una encuesta sobre actividad físico recreativa para adulto mayor, construida adaptando información por parte de la investigadora, tutora, variables del cuestionario de Actividad Física de Zutphen (Capersen, Bloemberg, Saris, Merrit y Kromhout, 1991); el cual reporta una validez de 0.78 y una confiabilidad de 0.89 y del cuestionario modificado de Baecke de adultos mayores (Voorrips, Ravelli, Dongelmans, Deurenberg, y Van Staveren, 1991) con una validez de 0.61 ( $p<0.05)$ y una 
confiabilidad de $0.93, \mathrm{p}<0.05$. En ésta encuesta se indagó sobre el tipo de actividad, preferencia y tiempo de las actividades. Además se contó con la participación de tres encuestadores a los cuáles se les dio la capacitación respectiva.

Procedimiento: El estudio de la perspectiva subjetiva de la calidad de vida que presentan los adultos mayores de 60 a 75 años de edad de 21 centros diurnos a nivel nacional, es una investigación ex-post facto exploratorio-diagnóstica según. Se realizó en primera instancia una revisión y selección de posibles centros a trabajar, con el correspondiente permiso y en los cuales se dio a conocer los propósitos y la colaboración necesaria para dicha investigación. Luego se procedió una vez aceptada la propuesta oficialmente, a capacitar durante una semana a los encuestadores en el uso de los instrumentos para la recolección de datos. La perspectiva subjetiva de calidad de vida, se determinó mediante cuatro variables: autonomía, soporte social, salud mental y actividad físico recreativa. Las tres primeras serán medidas por el cuestionario de calidad de vida INISA y la cuarta por la encuesta sobre actividad física para adulto mayor. La aplicación de los instrumentos se realizó con la visita personal de la investigadora en compañía de dos colaboradores previamente capacitados para la aplicación de los instrumentos a los sujetos seleccionados, además del apoyo correspondiente del coordinador de cada centro diurno. La duración del proceso de recolección de datos por sujeto encuestado fue de aproximadamente 20 minutos.

Análisis estadístico: El tratamiento estadístico de las variables a estudiar se realizó con el paquete estadístico SPSS y se aplicó el promedio (X), desviación estándar (S). También se aplicó el análisis de correlación $\mathrm{r}$ de Pearson para determinar la relación entre la autonomía, soporte social, salud mental (depresión, estado mental) y actividad física (frecuencia de la actividad, duración, disfrute y edad) y el coeficiente de determinación $\left(\mathrm{r}^{2}\right)$ para cada $\mathrm{r}$. También se procedió al cálculo de la Chicuadrada con el fin de determinar si existían diferencias en los motivos para realizar actividad física y en los beneficios que aporta esta actividad ligados al género de acuerdo a la encuesta sobre actividad físico recreativa.

\section{RESULTADOS}

A continuación se presentan los resultados obtenidos en el presente estudio.

Tabla 1. Resumen de promedios y desviaciones estándar de variables de calidad de vida y edad en adultos mayores costarricenses, según el género

\begin{tabular}{ccc}
\hline VARIABLE & Hombres (n:60) & Mujeres (n:92) \\
\hline Autonomía & $12.21 \pm 2.94$ & $11.93 \pm 2.52$ \\
Soporte Social & $2.96 \pm 1.72$ & $3.07 \pm 1.64$ \\
Depresión & $15.58 \pm 3.59$ & $17.13 \pm 4.11$ \\
Estado Mental & $21.71 \pm 6.06$ & $21.69 \pm 4.86$ \\
Frecuencia Actividad Física R. & $3.13 \pm 1.61$ & $3.01 \pm 1.40$ \\
Duración Actividad Física R. & $2.26 \pm 1.11$ & $2.21 \pm 0.73$ \\
Edad & $69.86 \pm 4.66$ & $69.97 \pm 4.11$ \\
\hline
\end{tabular}

En la tabla 1 se puede observar que tanto en hombres como en mujeres el promedio de autonomía fue similar; considerando la escala de calificación según INISA (1994), ambos se clasificaron como independientes en cuanto a esta variable. Por otra parte, en cuanto al soporte social, el promedio obtenido por los hombres y las mujeres estuvo ubicado en el puntaje intermedio de la escala, por tanto, el puntaje en ambos grupos fue regular. En lo que respecta a la depresión en el caso de las mujeres fue superior al de los hombres; sin embargo de acuerdo al puntaje obtenido, ambos se clasificaron con depresión leve. Se puede apreciar cómo los sujetos, tanto hombres como mujeres, tuvieron un promedio de estado mental que les ubicó en la categoría de trastornos depresivos y alteraciones cognitivas (según INISA, 1994). Con respecto a la frecuencia semanal de actividad física se puede observar que en ambos casos era entre 3 y 4 veces a la semana. Con respecto a la 
duración de la actividad física recreativa diaria se apreció que tanto hombres como mujeres tienden a hacer actividad física más de una hora diaria pero menos de una y media.

Por otra parte entre las variables calidad de vida y actividad física semanal en hombres, se presentó una correlación significativa entre autonomía y soporte social $(r=.28 ; p<0.05$; coeficiente de determinación $\mathrm{r}^{2}=7.84 \%$ de varianza compartida); entre autonomía y estado mental $(r=.27 ; p<0.05$; $\mathrm{r}^{2}=7.29$ ). Se presentó además una correlación significativa entre autonomía y actividad física semanal $\left(\mathrm{r}=.33 ; \mathrm{p}<0.05 ; \mathrm{r}^{2}=10.89 \%\right)$. Es decir que entre mayor frecuencia en la actividad física mayores niveles de autonomía presentó el adulto mayor. Además en la matriz de correlaciones de las variables calidad de vida y actividad física semanal en mujeres; se presentó una correlación significativa entre autonomía y soporte social $(\mathrm{r}=.24 ; \mathrm{p}<0.05$; el coeficiente de determinación $\mathrm{r}^{2}=5.76 \%$ de varianza compartida).

Se presentó además una correlación significativa entre autonomía y frecuencia de la actividad física $\left(\mathrm{r}=.24 ; \mathrm{p}<0.05 ; \mathrm{r}^{2}=5.76 \%\right.$ de varianza compartida) y entre estado mental y actividad física semanal $\left(\mathrm{r}=.23 ; \mathrm{p}<0.05 ; \mathrm{r}^{2}=5.29 \%\right)$. Es decir que entre mayor frecuencia en la actividad física mayores niveles de autonomía y salud mental presentaron. En lo que respecta a la matriz de correlaciones de las variables calidad de vida horas diarias de práctica o actividad física en hombres; se presentó una relación significativa entre autonomía y soporte social $\left(r=.28 ; \mathrm{p}<0.05 ; \mathrm{r}^{2}=7.84 \%\right)$; entre autonomía y estado mental $\left(r=.27 ; p<0.05 ; r^{2}=7.29 \%\right)$. Se presentó relación significativa entre autonomía y horas diarias de actividad física $\left(\mathrm{r}=.28 ; \mathrm{p}<0.05 ; \mathrm{r}^{2}=7.84 \%\right)$ y entre el soporte social y horas diarias de actividad física $\left(\mathrm{r}=.27 ; \mathrm{p}<0.05 ; \mathrm{r}^{2}=7.29 \%\right)$. Es decir que entre mayor tiempo de actividad física mayores niveles de autonomía y soporte presentó el adulto mayor. Matriz de correlaciones de las variables calidad de vida horas diarias de práctica o actividad física en mujeres; se presentó una relación significativa sólo entre autonomía y soporte social $\left(r=.24 ; \mathrm{p}<0.05 ; \mathrm{r}^{2}=5.76 \%\right)$.

Gráfico 1. Gráfico comparativo del motivo para realizar actividad físico recreativa en adultos mayores

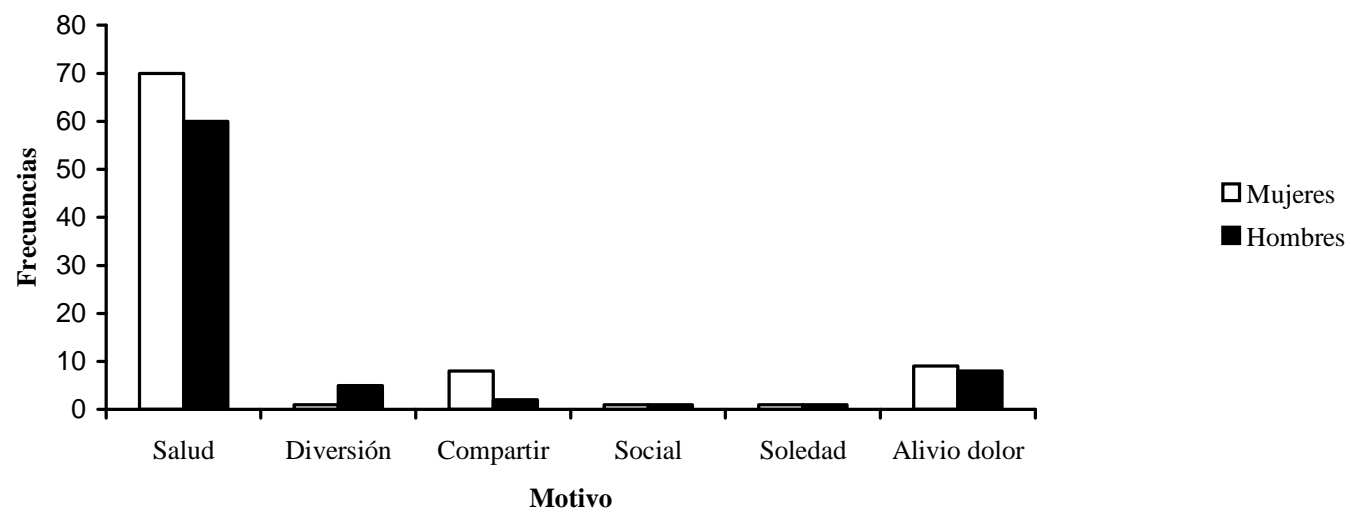


En cuanto al rubro motivo para hacer la actividad física no se encontró diferencias significativas $\left(\mathrm{X}^{2}=4.59, \mathrm{p}>0.05\right)$ de acuerdo al análisis estadístico; por lo tanto hombres y mujeres reportaron motivos similares y coincidieron en señalar principalmente la salud para hacer actividad física.

Gráfico 2. Gráfico comparativo de beneficios percibidos por realizar actividad físico recreativa en adultos mayores

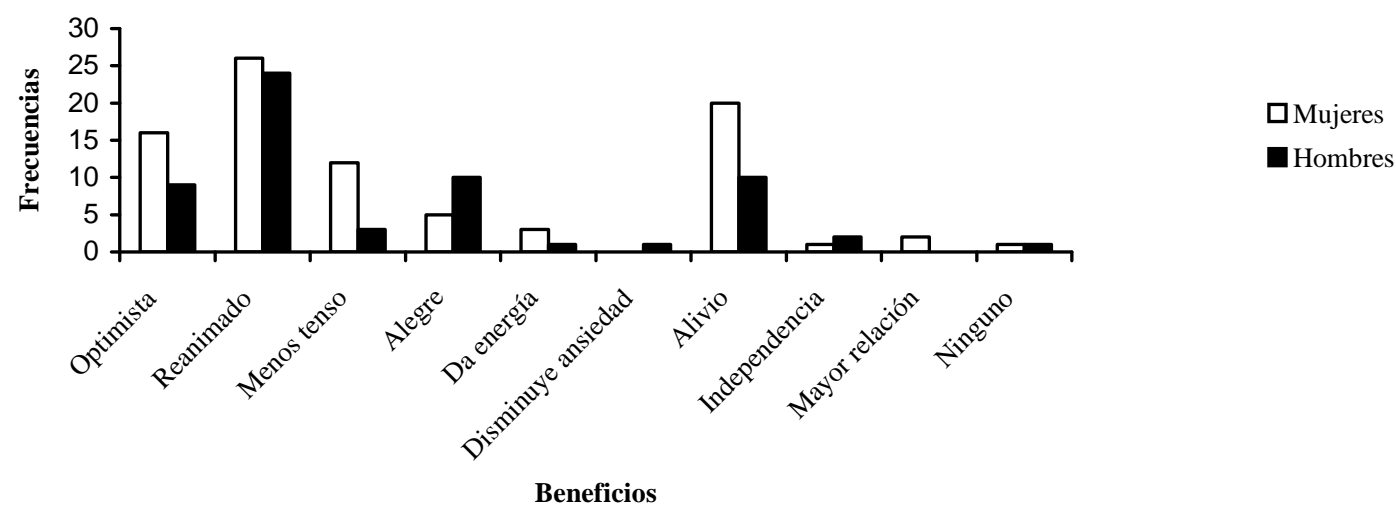

De acuerdo con el análisis estadístico, se obtuvo una $\mathrm{X}^{2}=22.49, \mathrm{p}<0.05$ lo cuál indicó que los hombres y las mujeres percibieron beneficios similares en la actividad física. Los hombres expresaron que la actividad física los hacía sentirse más reanimados y sienten que se alivian los dolores y las mujeres por lo general expresaron que la actividad física les hacía sentirse menos tensas, más alegres y con energía.

\section{DISCUSIÓN}

El propósito del presente estudio fue conocer la relación entre las variables autonomía, soporte social, salud mental (depresión y estado mental) y actividad física (frecuencia semanal, tiempo diario de la actividad, disfrute), considerando diferencias de género y edad, determinar el motivo principal para realizar la actividad física y el beneficio de acuerdo al género.

Por lo tanto, y de acuerdo con los resultados se determinó que la relación entre todas las variables de calidad de vida y la actividad física semanal tanto en hombres como en mujeres presentaron relaciones significativas entre autonomía y la actividad física semanal; es decir, que entre más actividad física hacían por semana, mejores niveles en autonomía presentaban; destacándose que al tener más personas asistentes a centros diurnos son más independientes (Céspedes, 1987) y colaboradores siendo congruente con el estudio de Rose Medical Center y el Rose Health Care System en Colorado (2001) de que los adultos mayores al estar involucrados en programas de actividad física recreativa en los centros diurnos se aumentan los niveles de movilidad e independencia y autoconcepto lo que contribuye a un aumento en los índices de apreciación de vida y de actividad física. Además de que la actividad física metódica y programada genera según Ramos (1992), mejoras en la producción de placer y bienestar corporal y mental, ya sea en la conservación y consecución de independencia y autonomía tanto física como psíquica. Se presentó además relaciones significativas entre autonomía y soporte social en ambos géneros; destacándose que entre más autónomos sean mayor Soporte Social presentan, siendo congruente con el estudio de Céspedes (1987), en donde las personas al ser 
asistentes a centros diurnos, tienen mayor grado de independencia y al ser miembros dentro de las familias y comunidades exigen en éste mayor participación, opinión y toma de decisión, lo que favorece al desarrollo de su independencia según Quirós (1996); siendo además congruentes estos resultados con los expuestos por Rojas (1999) en su investigación sobre calidad de vida y autonomía, en general los adultos perciben un sentimiento de bienestar, fortalecido por ser personas independientes y funcionales; en los hombres se presentó además relación significativa entre autonomía y estado mental; tomando en cuenta que son personas más participativas y activas según Villalobos (1989), lo que les permite según Quirós (1998), en su salud mental enfrentar de manera exitosa y satisfactoria su vida.

Además se encontró relación significativa entre el estado mental y actividad física semanal en las mujeres. Es decir que entre mayor frecuencia en la actividad física mayores nivelen la salud mental presentaron siendo congruente con lo apuntado por Ramos (1992) acerca de que la actividad física metódica y programada genera mejoras en la producción de placer y bienestar tanto corporal como mental.

En lo que respecta a la relación de todas las variables con las horas diarias de práctica en hombres, se presentaron relaciones significativas entre autonomía y horas diarias de práctica física y entre soporte social, horas diarias de práctica, es decir que entre mayor duración en la práctica de actividad física mejores niveles de autonomía y soporte social presentaron; destacándose que entre más independientes, colaboradores; los adultos mayores asistentes en centros diurnos son más participativos y activos; con lo que se justifican la disposición y el tiempo para realizar la actividad física (Villalobos,1989); además de que se compara la actividad física con la experiencias ascética, caracterizada ésta por aquellas actividades que requieren de un entrenamiento largo y cansado, condicionamiento pero finalmente presentan gratificaciones a largo plazo según el modelo multidimensional propuesto por Kenyon (1980).

Asimismo, se presentó relación significativa entre autonomía y soporte social en ambos géneros los adultos mayores asistentes en centros diurnos son más participativos y activos (Villalobos, 1989) y al ser miembros dentro de las familias y comunidades tienen mayor participación, opinión y toma de decisión, según Quirós (1996). Por otra parte, en los hombres se presentó relación significativa entre autonomía y estado mental; al ser personas independientes son más participativos y activos según Villalobos (1989) lo que les permite según Quirós (1998) en su salud mental enfrentar de manera exitosa y satisfactoria su vida.

Por otra parte, se destacó que con respecto a la depresión, en las mujeres fue superior al de los hombres, siendo congruente con Stephens (1988), el cuál menciona que la depresión y la ansiedad es más frecuente en las mujeres; pudiendo ser producto de las actitudes de la familia, comunidad y sociedad en general como rechazo, sobreprotección o abuso contra ellas. Sin embargo, hay que mencionar que en cuanto a los resultados tanto hombres como mujeres se clasificaron con depresión leve, siendo congruente con lo apuntado por Quirós (1996), acerca de que la depresión en el adulto mayor es una de las condiciones más frecuentes y de mayor cuidado; además de que se rechaza el mito de que la depresión es una enfermedad característica de la mujer y comprender que según Marín y col (2000), los factores que contribuyen al desarrollo de la depresión no dependen del género sino de la problemática social en el que esté inserto (abuso y abandono).

Se determinó que los adultos mayores tienen cierto grado de deterioro tanto físico como mental; sin embargo, los sujetos hombres y mujeres con mayores niveles de actividad física reportados tanto en frecuencia como en duración diaria; tienden a un estado mejor; lo que indica que a pesar de que continúa el deterioro normal, la actividad física en estas poblaciones es fundamental para mantener un adecuado nivel de calidad de vida congruente con los estudios de Hernández (2000), Esquivel y Jiménez (1997), Fugisawa, (1994), entre otros. 
En lo que respecta al motivo para realizar actividad física tanto hombres como mujeres coinciden en señalar la salud, siendo congruente con los resultados del estudio sobre actividad física y estado emocional (Bolaños y Mora, 1999), asimismo es congruente con Schwenkmezger (1993, citado en Ureña, 1996), el cuál destaca que el motivo salud tienen un valor preponderante considerando la parte somática y la dimensión psicosocial. Siendo además congruente con Idespo (1985), ya que en general los adultos mayores manifestaron interés en actividades productivas; y la motivación que genera la actividad físico recreativa aumentaron en ellos el autoconcepto, la autoestima y las emociones, distrayéndose al hacer algo en lo que se sentían útiles y ocupados como en el caso de los sujetos del presente estudio.

Finalmente, en cuanto a los beneficios o disfrute de la actividad física los valores para ambos géneros fueron elevados, les aliviaban los dolores y en le caso de las mujeres les hacía sentirse menos tensas, más alegres y con energía, siendo congruente por lo planteado por Ureña y Delgado (1998), sobre la percepción subjetiva de la persona mayor en relación con el ejercicio físico, mostrando una percepción más positiva de sí mismos, un mayor disfrute. Asimismo, la actividad física ha demostrado notables mejoras en la producción de placer y bienestar corporal y mental (Ramos, 1992); por lo que coinciden los resultados encontrados en este estudio.

\section{CONCLUSIONES}

En síntesis se puede concluir que la calidad de vida (autonomía, soporte social, salud mental, actividad física recreativa) difiere en lo que respecta al género. La depresión geriátrica presentó diferencias significativas en cuanto al género siendo superior en las mujeres. La salud en ambos fue el principal motivo para realizar la actividad física recreativa y los beneficios fueron sentirse reanimados, aliviados; más alegres y con energía. Los adultos mayores con mayores niveles de actividad física tanto en frecuencia como en duración diaria tienden a tener un mejor nivel de calidad de vida.

\section{REFERENCIAS}

Abrante, C., Brito, O. y García, C. (1996) "El deporte en edad avanzada". Programa de actividades físicas y deportivas para personas mayores. Gran Canaria.: Editorial Nogal.

AGECO (1988). Algunos consejos prácticos en la tercera edad. San José

Aztarain, F; Beorlegui, M. (1994). Sesenta minutos a la semana para la salud. Archivo medicina del Deporte. X (41), 49-54.

Capersen, C. J.; Bloemberg, B. P. M.; Saris, W. H. M.; Merrit, R. K. y Kromhout, D (1991). Physical activity questionnaires for older adults. Journal Sport Medicine. 29 (6), 141145 .

Céspedes, A. y col (1987). Influencia de los factores socioeconómicos en la pérdida de autonomía de los adultos mayores costarricenses entre los 65 y 80 años. Programa de Investigación sobre el envejecimiento. San José, Costa Rica: Editorial UCR.

Díaz, P., Puente, G y García, T. (1994, Enero-Febrero). "Resultados de la aplicación de una escala de valoración socio familiar en atención primaria". Revista Española de Geriatría y Gerontología. 29, 239-245

Esquivel R., Jiménez, F. (1997) Evaluación subjetiva de bienestar en ancianos. Disponible en: http://www.iztacala.unam.mx/coloquio/saludcartel/145.html. Fecha de visita: (06-092000). 
Faden, R. y Germán, P. (1994). Calidad de vida. Consideraciones en geriatría. Clínica de Medicina Geriátrica. México, D. F.: Editorial Interamericana.

Febrer, A (1996) Cuerpo, dinamismo y vejez. Barcelona, España: Editorial INDE.

Fernández, R. (1994) Evaluación e intervención psicológica en la vejez. España: Editorial Martínez Roca.

Fujisawa, M. (1994, Octubre). Comparative study of quality of life in the elderly between in Kahoku and in Yaku. Kochi Medical School Journal. Japón .Oct. 31 (1) 10, 790-799.

Garay, F (1996). Bienestar Psicológico del adulto mayor. Madrid, España: Editorial Herder.

George, L.; Bearon, L (1980). Quality of life in older persons: meaning and measurement. New York: Editorial Human Sciences Press.

Giusti, L. (1991). Calidad de vida, estrés y bienestar. San Juan, Puerto Rico: Editorial Psicoeducativa.

González, A. (1994). Aprendamos a envejecer sanos. Manual de auto cuidado y salud en el envejecimiento. 3ra. Edición. México.

Hernández, Z. (2000). Elaboración de un índice de calidad de vida para personas de la tercera edad. México.

INISA, (1994). Validación de un instrumento para la medición de la calidad de vida de personas de 60 años y más. Programa de Investigación sobre el envejecimiento. San José, Costa Rica: Editorial UCR.

Leturia, F. (1998). Jubilación y calidad de vida. Geriátrica Gerontológico 33, 9-16.

Levi y Anderson (1980). La tensión psicoemocional. Población, ambiente y calidad de vida. España: Editorial Vitoriagasteiz.

Quirós, M. (1996). Elaboración de un modelo de salud comunitaria Geronto-Geriátrico, basado en la calidad de vida. Tesis para optar por el grado de magíster en Gerontología. San José: UCR.

Ríos, L., Ríos, I. y Padial, P. (2000) "La actividad física en la Tercera Edad". Digital 5 (18 febrero), 1-2.

Rojas, M. (1997). Bienestar general subjetivo y depresión en ancianos crónicos: un estudio de casos. Tesis para optar por el grado de Magíster en Gerontología. San José, Costa Rica: Editorial UCR.

Rojas, L. (1999). Calidad de vida y autonomía en personas mayores. Tesis para optar por el grado de Magíster en Gerontología. San José, Costa Rica: Editorial UCR.

Roy, A. y Fitz, P. (1996 Marzo). Social support, household composition, and health behaviors as risk factors for year mortality in an urban elderly cohort. Journal of Applied Gerontology. 15(1), 73-78.

Stephens, R. (1988). Actas Congreso Científico Olímpico. Actividad Física Adaptada, Psicología y Sociología. Bible, S. 
Thomas, J.R. \& Nelson, J.K. (1990) Research Methods in Physical Activity. Illinois: Editorial Human kinetics books.

Timiras, PS. (1996). "Physiology of aging: standards for age-related functional competence". In Greger R, Windhorst U (Eds): Comprehensive Human Physiology. Vol.2 BerlinHeidelberg: Springer-Verlag, 2391-2404.

Ureña, P. (1996). Práctica deportiva y salud. Perspectiva Psicológica. Heredia, Costa Rica: Editorial UNA.

Villalobos, D. (1989). Problemas de salud que enfrenta la población de la tercera edad en la ciudad de Heredia. Tesis de Magíster Scientiae. UCR. San José, Costa Rica.

Voorrips, L. E.; Ravelli, A. C. J.; Dongelmans, P. C. A.; Deurenberg, P.y Van Staveren, W.A. (1991). Physical activity questionnaires for older adults. Journal of Sports Medicine. 29 (6), 117-121.

Fecha de recepción del artículo: 04 de agosto del 2004.

Fecha de aceptación del artículo: 23 de setiembre del 2004.

Fecha de publicación del artículo: 24 de setiembre del 2004. 